\title{
Análisis del componente cultural en libros de texto de francés destinados al tercer ciclo de Educación Primaria
}

\section{Analysis of the cultural component in textbooks for teaching French in the third stage of Primary Education}

\author{
ELISA GIL RUIZ \\ eli.gil@um.es \\ CARMEN SOTO PALLARÉS \\ carsoto@um.es \\ Universidad de Murcia, España
}

\section{Resumen:}

En la didáctica de lenguas extranjeras, el componente cultural se establece como un elemento de suma importancia para el desarrollo de la competencia comunicativa del alumnado. Por este motivo, la adquisición de la lengua francesa debe desarrollarse en un marco ecléctico que favorezca la integración de contenidos tanto lingüísticos como culturales, siendo necesaria la inclusión del componente cultural en los materiales curriculares. Desde niveles iniciales, la dimensión cultural, que subyace a todo acto comunicativo, debe estar presente en la enseñanza de lenguas, razón por la que hemos emprendido el análisis de los contenidos culturales presentes en los libros de lengua francesa empleados en el tercer ciclo de la Educación Primaria, para determinar si su tratamiento didáctico es óptimo y si se ajusta a las orientaciones de los enfo-

\begin{abstract}
:
In Foreign Languages Teaching, the cultural component is of paramount importance for the development of students' communicative competence. For this reason, the acquisition of the French language must be developed within an eclectic framework promoting the integration of both linguistic and cultural contents; this way, it is necessary to include the cultural component in educational resources. From the initial levels, the cultural dimension underlying any communicative act must be taken into account when teaching languages. That is the reason why the cultural contents in textbooks for teaching French in the third cycle of Primary Education have been analysed, to determine the quality of its pedagogical treatment and their suitability following the orientations of the leading methodological approaches in foreign language teaching.
\end{abstract}

1 Dirección para correspondencia (correspondence address):

Elisa Gil Ruiz. Dpto. de Didáctica de la Lengua y la Literatura. Facultad de Educación. Universidad de Murcia. Campus de Espinardo. 30100, Murcia (España). 
Análisis del componente cultural en libros de texto de francés destinados al tercer ciclo de Educación Primaria

ELISA GIL RUIZ y CARMEN SOTO PALLARÉS

ques metodológicos predominantes en la didáctica de las lenguas extranjeras.

\section{Palabras clave:}

Lengua y cultura extranjeras; análisis de libros de texto.

\section{Key words:}

Foreign language and culture; textbook analysis.

\section{Résumé:}

Dans la didactique des langues étrangères, le composant culturel occupe une place importante dans le développement de la compétence communicative des élèves. C'est pour cette raison que l'acquisition de la langue française doit se développer dans un cadre éclectique favorisant l'intégration des contenus aussi bien linguistiques que culturels, en n'omettant pas l'inclusion du composant culturel dans les matériels curriculaires. À partir des niveaux basiques, la dimension culturelle, qui est sous-jacente à tout acte communicatif doit être présente dans l'enseignement des langues. C'est pour cela que nous avons entrepris une analyse des contenus culturels présents dans les manuels scolaires en langue française utilisés dans le troisième cycle de l'Éducation Primaire, afin de déterminer si son emploi didactique est optimal et s'il s'adapte aux orientations des approches méthodologiques prédominantes en didactique des langues étrangères.

\section{Mots-clés:}

Langue et culture étrangères; analyse des manuels scolaires.

Fecha de recepción: 16-12-2013

Fecha de aceptación: 14-5-2014

\section{Introducción}

Actualmente, en el contexto de la didáctica de lenguas extranjeras, la idea de que el aprendizaje de un idioma conlleva la adquisición de los elementos culturales que éste tiene implícitos es comúnmente reconocida y aceptada. Existe una estrecha relación entre toda lengua y la cultura, que se resume de la siguiente manera: la lengua es reflejo y manifestación de una cultura formando parte de ésta y la cultura configura y determina el empleo de la lengua de una determinada comunidad.

En este sentido, Guillen Díaz (2002), ha puesto de relieve la relación de indisociabilidad, que existe entre toda lengua y la cultura, poniendo en relieve la siguiente idea:

"Lengua y cultura se nos presentan como un todo indisociable, pues a todo hecho de lengua subyace un hecho de cultura y porque todo hecho de lengua se estructura en función de una dimensión cultural." (Guillén Díaz, 2002:202) 
Normalmente, el estudio de una lengua requiere una interpretación del significado social y culturalmente establecido, y al mismo tiempo, el estudio de distintos aspectos culturales requiere una comprensión de los elementos lingüísticos empleados para su transmisión. Por tanto, en el ámbito educativo importa integrar la dimensión cultural en el proceso de aprendizaje de toda lengua extranjera, adquiriendo los elementos culturales de manera contextualizada, tal y como establece Guillen Díaz (2002):

"En la intervención pedagógica, el componente cultural debe ser abordado como el complemento de un conjunto de funciones y nociones prácticas que implica una lengua para decir y hacer en la vida cotidiana. Se trata de una visión del mundo extranjero que hay que descubrir por medio de la lengua, en contexto y en el seno de las interacciones entre los locutores que utilizan esa lengua." (Guillén Díaz, 2002:217)

De esta manera, la integración del componente cultural contribuye al desarrollo de la competencia comunicativa de los alumnos, siendo éste un elemento central del proceso de aprendizaje lingüístico.

\section{El tratamiento didáctico del componente cultural}

Tradicionalmente, en la didáctica de lenguas extranjeras, la lengua y la cultura se abordaban por separado como si se tratasen de realidades inconexas. Sin embargo, en la actualidad se ha generalizado la idea de la existencia de una íntima conexión entre la lengua y la cultura, por lo que en todo proceso de adquisición de cualquier idioma extranjero ambas deben desarrollarse de manera integrada porque son realidades indisociables. Además, el Enfoque Comunicativo y la Perspectiva Accional han influido positivamente en este cambio didáctico, ya que en ambos se empieza a considerar el componente cultural como un elemento que determina el desarrollo de la competencia comunicativa de manera conjunta con los demás integrantes que la componen.

En la evolución que ha experimentado el tratamiento didáctico del componente cultural, vamos a constatar la sustitución progresiva del enfoque informativo hacia al empleo del enfoque formativo, que es el más recomendado en la actualidad. En ambos enfoques, la percepción de 
Análisis del componente cultural en libros de texto de francés destinados al tercer ciclo de Educación Primaria

ELISA GIL RUIZ y CARMEN SOto Pallarés

la cultura ha ido también evolucionando desde una perspectiva formal hasta llegar al establecimiento de una cultura no formal, e incluso al establecimiento posterior del concepto de "Kultura con K".

De manera tradicional, en clase de lenguas extranjeras se desarrollaba una cultura con 'C mayúscula', también Ilamada 'Cultura Formal', integrada por las Ilamadas "instituciones" culturales, que abarcan la historia, la geografía, las artes (la literatura, la música...), la política, la religión, el folklore y la economía características todas ellas del país extranjero. Sirva como definición de Cultura Formal el perfil que le otorga Areizaga (2002):

“(...) una mezcla de datos históricos, políticos, geográficos, con información sobre los logros y producciones de personajes de prestigio, junto con lo más folklórico y pintoresco de los países donde se habla la lengua meta." (Areizaga, 2002:162).

El desarrollo de la Cultura Formal en clase de lenguas extranjeras aportaba al alumnado una gran cantidad de conocimientos y de informaciones sobre la cultura extranjera, que servía para engrandecer las ideas de los alumnos pero que en ningún momento les enseñaba a aplicar dichos contenidos en el desarrollo de intercambios comunicativos. Adviértase por ello que, si bien esta perspectiva puede ayudar a una mejor relación con dicha cultura, sin embargo no contribuye al desarrollo de la competencia comunicativa, que hace a los aprendices competentes a la hora de emplear la lengua en determinadas situaciones culturalmente condicionadas.

Con el desarrollo del enfoque formativo se aboga por el tratamiento de la cultura con 'c minúscula' o 'Cultura no Formal', constituida por las características culturales que definen a un grupo social, tales como los patrones adquiridos de conducta, las creencias y los valores, las costumbres, los hábitos, las actitudes, las tradiciones, las formas cotidianas de vida y el universo significativo de esa comunidad lingüística. En esta línea cabe citar la esclarecedora definición de 'Cultura no Formal' aportada por Areizaga, Gómez e Ibarra (2005):

"Una visión no formal de la cultura está centrada en los aspectos socioculturales de los hablantes de la lengua meta, más acorde con la forma de entender la lengua como herramienta de las relaciones sociales de una comunidad." (Areizaga, Gómez e Ibarra, 2005:28) 
En resumidas cuentas, este tipo de cultura que nosotros Ilamamos 'cultura no formal' abarcaría todos los conocimientos socioculturales necesarios para desenvolverse de forma adecuada en una situación comunicativa, por lo que deben ser introducidos en todo proceso de adquisición de una lengua extranjera.

A estas dos maneras de desarrollar la cultura en clase de lenguas extranjeras, (la formal y la no formal), Miquel y Sans (1992:18) añade el concepto de 'Kultura con $\mathbf{K}^{\prime}$, la cual se centra en la identificación social o cultural de un interlocutor para así poder actuar adecuadamente, adaptándose a ese interlocutor, cuando sus usos y sus costumbres difieren del estándar cultural, por lo que no son compartidos por todos los hablantes, tales como los argot de ciertos sectores de la población.

De acuerdo con estas dos autoras, estos tres tipos de culturas no son sectores estancos, ya que, en bastantes ocasiones, todas ellas "van a revertir en el cuerpo central de la cultura, engrosando la zona de lo compartido por todos." (Miquel y Sans, 1992:19).

\section{Objetivo de investigación}

Teniendo en cuenta la necesidad de favorecer la integración de la lengua y la cultura en la adquisición de una lengua extranjera, hemos considerado que dicha unión debe reflejarse en el desarrollo de los libros de texto empleados para el aprendizaje de un idioma extranjero. Así que, partiendo de esta premisa, el desarrollo de nuestra investigación pretende analizar el tratamiento didáctico otorgado al componente cultural en los libros de texto, empleados para la adquisición del francés, como segunda lengua extranjera, en el tercer ciclo de la Educación Primaria, pertenecientes a la editorial Santillana. En síntesis, nuestro propósito es determinar si el desarrollo cultural presente en esta editorial es significativo y tiene en cuenta los preceptos legales en vigor.

\section{Metodología}

La metodología empleada para el desarrollo de esta investigación se enmarca en el paradigma cualitativo, empleando estrategias de tipo descriptivo e interpretativo, puesto que, pretendemos detectar y analizar 
Análisis del componente cultural en libros de texto de francés destinados al tercer ciclo de Educación Primaria

ELISA GIL RUIZ y CARMEN SOto Pallarés

las actividades que vehiculan elementos culturales que se encuentran presentes en los libros de francés pertenecientes a la editorial Santillana.

Previamente al análisis del componente cultural, nos hemos centrado en los aspectos generales que presentan cada uno de los libros estudiados, teniendo en cuenta los siguientes criterios:

- El nivel al que va dirigido cada libro.

- El número de páginas del libro y la cuantía de unidades didácticas que integra.

- La organización y la estructuración de los contenidos en el desarrollo de cada unidad didáctica, la cual está reflejada en el índice.

- La presencia de las competencias básicas, como un nuevo elemento curricular que deben introducir los libros de texto para estar en consonancia con las actuales leyes educativas (LOE).

- El enfoque metodológico adoptado en relación a la adquisición de una lengua extranjera, teniendo en cuenta las tendencias actuales, que se asientan en el eclecticismo, el cual aúna rasgos metodológicos del Enfoque Comunicativo, del Enfoque por tareas y de la Perspectiva Accional.

Para llevar a cabo el análisis de los elementos culturales detectados, vamos a emplear un instrumento de recogida de información, que es de elaboración propia, integrado por dos tipos de plantillas de análisis, cuya principal diferencia radica en la forma de recogida de los datos, ya que, ambas se asientan en las mismas variables, las cuales se establecen de manera resumida a continuación:

- La existencia de un apartado específico dedicado al componente cultural.

- Los temas culturales abordados en cada una de las unidades didácticas de cada libro de texto.

- La correspondencia entre los temas culturales destacados en el índice de contenidos y los realmente desarrollados en las unidades didácticas.

- La relación existente entre los temas desarrollados en los libros de texto y los bloques temáticos del MCERL (2002).

- El medio o soporte empleado para la transmisión de los contenidos culturales.

- El tipo de actividad que integra aspectos culturales.

- El porcentaje que suponen las muestras culturales con respecto 
a la totalidad de las actividades que presenta cada libro de texto.

- El tratamiento didáctico otorgado al componente cultural en cada uno de los libros, siempre teniendo en cuenta las características del enfoque informativo y del enfoque formativo e intercultural.

- El tipo de cultura vehiculado por la muestra cultural analizada, es decir, si se trata de una muestra cultural perteneciente a la cultura formal, no formal o a la 'Kultura con $\mathrm{K}^{\prime}$

- El desarrollo de las competencias básicas en las actividades culturales estudiadas.

\section{Resultados}

\subsection{Análisis de los aspectos generales de los libros de texto}

El análisis de los aspectos generales de cada uno de los dos libros de texto pertenecientes a la editorial Santillana nos muestra que ambos están destinados a alumnos principiantes que cursan el tercer ciclo de la Educación Primaria. El libro destinado a los alumnos de quinto curso, recibe el nombre de 'Vitamine 1' mientras que el propuesto para sexto curso tiene por título 'Vitamine 2'. Ambos libros están orientados a alumnos que se encuentran en un nivel A1, según los niveles establecidos por el MCERL (2002).

A pesar de que ambos libros presentan una estructura similar y una organización de los contenidos análoga, cada uno no presenta el mismo número de unidades didácticas: el libro 'Vitamine 1' está estructurado en torno a 8 unidades didácticas desarrolladas a lo largo de 64 páginas, mientras que el de 'Vitamine 2' está organizado alrededor de 9 unidades didácticas desarrolladas, también, a lo largo de 64 páginas. Estas 9 unidades didácticas del libro 'Vitamine 2' no están numeradas del 1 al 9, sino que la primera es la unidad 0 porque es la de repaso de los conocimientos previos adquiridos durante el curso anterior con el uso del libro 'Vitamine 1'. El resto de las unidades didácticas están numeradas del 1 al 8. Además, cada libro incluye tres suplementos culturales, situados cada dos unidades didácticas, que recibe el nombre de "France Magazine".

Por su parte, cada uno de los dos libros presenta una organización similar de los contenidos en el índice, ya que, cada unidad didáctica está estructurada siguiendo los siguientes apartados: 'Communication, 
Análisis del componente cultural en libros de texto de francés destinados al tercer ciclo de Educación Primaria

ELISA GIL RUIZ y CARMEN SOto Pallarés

Grammaire, Vocabulaire, Phonétique, Civilisation, Tâche finale et Compétences Clés.' Todas estas secciones y su desarrollo están recogidos en el índice de cada libro de texto, en el que se especifican los contenidos trabajados en cada unidad didáctica. Obsérvese que en el último apartado de ambos índices se presentan las competencias básicas que cada unidad didáctica desarrolla de manera específica. Además, la editorial destaca que en todas las unidades didácticas se encuentran todas las competencias básicas desarrolladas de forma implícita y transversal. De esta manera, esta editorial contempla el desarrollo de las competencias básicas, que es el nuevo elemento curricular que se establece como consecuencia de la promulgación de la Ley Orgánica de Educación (2006) y de las disposiciones legales que la desarrollan.

Tras el índice en el que se presentan los apartados de cada unidad didáctica, nos encontramos con la presentación de los personajes que van a intervenir a lo largo del libro. También se establecen los pictogramas y los diferentes tipos de actividades que se repiten en cada unidad didáctica. El recurso a los pictogramas al principio del libro nos hace pensar en el desarrollo de aspectos metodológicos propios de los enfoques actuales, tal como el empleo de ilustraciones para facilitar la comprensión del mensaje para no utilizar así la lengua materna.

Siguiendo con la estructura general de ambos libros, al final de la última unidad didáctica se encuentra la trascripción de todas las actividades de escucha que no presentan la letra en el desarrollo del libro. De esta manera, aquellas actividades de escucha cuya letra no se encontraban en la unidad didáctica correspondiente, como por ejemplo ocurre con algunas canciones, están en las hojas de trascripción situadas al final del libro.

Por este motivo, en este libro de texto todas las actividades de escucha que no presenten en su desarrollo la letra y que sean analizadas por su contenido cultural, serán consideradas una muestra desarrollada tanto en soporte texto como audio, puesto que, los alumnos podrán tener acceso a la trascripción escrita de dicha actividad.

El uso de cada uno de los soportes estará en función de la utilización que el docente decida hacer del libro y del objetivo perseguido en cada momento. Dichas trascripciones se encuentran organizadas según la unidad didáctica y la página en las que se halla cada actividad.

Para finalizar la descripción de la estructura general de los libros de esta editorial, tras las trascripciones, en el libro 'Vitamine 1 ' nos encon- 
tramos con un mini diccionario del léxico trabajado en cada unidad didáctica, el cual aparece acompañado del dibujo correspondiente para favorecer así la comprensión de dicho vocabulario sin tener que recurrir a la traducción. Este mini diccionario cierra este libro de texto. En cambio, en el libro 'Vitamine 2' se encuentra un resumen gramatical que engloba todos los contenidos gramaticales trabajados en las distintas unidades didácticas, el cual está acompañado de dibujos que favorecen la comprensión del alumnado sin tener que recurrir a la traducción.

Tras presentar los apartados en los que se estructuran ambos libros, nos vamos a centrar en el desarrollo de las orientaciones metodológicas, algunas de las cuales ya han sido detectadas anteriormente. Cabe comenzar por el hecho de que presentar ilustraciones que favorezcan la comprensión del alumnado está en concordancia con los enfoques metodológicos actuales, tales como el Enfoque Comunicativo, el Enfoque por tareas y la Perspectiva Accional. Además, en esta misma línea metodológica, se encuentra el propósito de no emplear la lengua materna ni la traducción, para que la lengua extranjera se establezca como el principal código de comunicación. Uno de los aspectos que puede favorecer este aspecto metodológico es la explicación de las actividades en lengua extranjera, como establece esta editorial, la cual presenta siempre las actividades por medio de instrucciones en francés.

Otro aspecto a destacar es la presencia de la realización de una tarea presente al final de cada unidad didáctica, que está directamente en relación con los enfoques didácticos empleados en la actualidad, los cuales son el Enfoque por tareas y la Perspectiva Accional, que complementan el Enfoque Comunicativo. Además, el MCERL (2002) destaca que en el aprendizaje de una lengua extranjera el alumno debe adquirir la capacidad para realizar tareas que no sean exclusivamente lingüísticas, sino también sociales, ya que, éstas les serán útiles en el desarrollo de intercambios comunicativos.

A este respecto, los dos libros de esta editorial presentan las secciones 'Activité Brico', 'Activité écriture' o 'Activité création', todas ellas con un carácter lúdico, en las que, tras el desarrollo de la unidad didáctica, los alumnos deben elaborar algún trabajo que permita la puesta en práctica de los contenidos trabajados anteriormente, utilizando para ello el formato de tarea final. Sin embargo, hay que señalar que a estas tareas les falta una orientación social, ya que son actividades planteadas a nivel individual, aunque luego deban ser expuestas ante el grupo clase. Por 
Análisis del componente cultural en libros de texto de francés destinados al tercer ciclo de Educación Primaria

ELISA GIL RUIZ y CARMEN SOto Pallarés

otro lado, cabría destacar que estas tres secciones presentan la ventaja de involucrar al alumnado de manera directa y activa en su aprendizaje, convirtiéndose así en el protagonista de todo el proceso, lo que contribuye también al desarrollo de las orientaciones metodológicas pertenecientes a los enfoques actuales.

\subsection{Análisis de los elementos culturales en los libros de francés de la editorial Santillana}

Tras el desarrollo de los aspectos generales de los libros pertenecientes a la editorial Santillana, vamos a detallar el desarrollo del componente cultural en cada uno de los libros de esta editorial, destinados a la adquisición de la lengua francesa en el tercer ciclo de la Educación Primaria.

En las distintas unidades didácticas de ambos libros existe una sección explícita dedicada a la cultura, que recibe el nombre de 'Civilisation'. En esta sección hemos encontrado una parte de las muestras culturales analizadas, las cuales están desarrolladas de manera aislada con respecto a los contenidos lingüísticos. También hay que destacar el suplemento cultural introducido por esta editorial cada dos unidades didácticas, bajo el nombre de 'France Magazine', en el que se desarrollan contenidos culturales aislados del aprendizaje lingüístico, ya que este apartado es presentado como si fuera un compartimento estanco y separado.

No obstante, la existencia de estas dos secciones específicas no ha impedido detectar diversas muestras culturales en la totalidad de cada unidad didáctica. Algunas de las actividades analizadas, aún sin pertenecer al apartado de 'Civilisation' o al suplemento cultural, han sido analizadas a lo largo de nuestro estudio, precisamente por su contenido cultural. En este caso, las actividades culturales se desarrollan de manera integrada con el aprendizaje lingüístico.

Este análisis comparativo revela que los temas abordados en esta sección son diferentes en cada libro, presentando siempre aspectos culturales familiares para los alumnos en una progresión que va de lo simple a lo complejo y de lo próximo a lo lejano, lo que les permite establecer conexiones con su propia experiencia, para así involucrarse activamente en su aprendizaje. Los contenidos tratados en esta sección se establecen en el índice de cada libro, presentados en la tabla 1: 
Tabla 1. Temas culturales tratados en la sección de 'Civilisation' de los libros de la editorial.

\begin{tabular}{|c|c|c|c|}
\hline \multicolumn{4}{|c|}{ EDITORIAL SANTILLANA } \\
\hline \multicolumn{2}{|c|}{ VITAMINE 1} & \multicolumn{2}{|c|}{ VITAMINE 2} \\
\hline Unidad didáctica 1 & Salutations & Unidad didáctica 0 & Collège: rentrée \\
\hline Unidad didáctica 2 & $\begin{array}{l}\text { Situation sco- } \\
\text { laire }\end{array}$ & Unidad didáctica 1 & $\begin{array}{l}\text { Collège: rentrée, } \\
\text { matières, activités }\end{array}$ \\
\hline $\begin{array}{l}\text { Suplemento: Fran- } \\
\text { ce Magazine } 1\end{array}$ & $\begin{array}{l}\text { La France : } \\
\text { histoire et } \\
\text { actualités }\end{array}$ & Unidad didáctica 2 & $\begin{array}{c}\text { Collège: horaires, } \\
\text { tenue vestimen- } \\
\text { taire }\end{array}$ \\
\hline Unidad didáctica 3 & Prénoms usuels & $\begin{array}{l}\text { Suplemento: Fran- } \\
\text { ce Magazine } 1\end{array}$ & $\begin{array}{l}\text { Géographie, his- } \\
\text { toire et actualité }\end{array}$ \\
\hline Unidad didáctica 4 & Loisirs & Unidad didáctica 3 & Prénoms usuels \\
\hline $\begin{array}{l}\text { Suplemento: Fran- } \\
\text { ce Magazine } 2\end{array}$ & Parc Naturels & Unidad didáctica 4 & Loisirs \\
\hline Unidad didáctica 5 & Vêtements & $\begin{array}{l}\text { Suplemento: Fran- } \\
\text { ce Magazine } 2\end{array}$ & $\begin{array}{l}\text { Cuisine françai- } \\
\text { se: recette de } \\
\text { la mousse au } \\
\text { chocolat }\end{array}$ \\
\hline Unidad didáctica 6 & Politesse & Unidad didáctica 5 & Écologie \\
\hline $\begin{array}{l}\text { Suplemento: Fran- } \\
\text { ce Magazine } 3\end{array}$ & Villes et sports & Unidad didáctica 6 & Habitudes \\
\hline Unidad didáctica 7 & $\begin{array}{l}\text { Desnos un } \\
\text { poète }\end{array}$ & $\begin{array}{l}\text { Suplemento: Fran- } \\
\text { ce Magazine } 3\end{array}$ & $\begin{array}{l}\text { Journée d'une } \\
\text { jeune française }\end{array}$ \\
\hline \multirow[t]{2}{*}{ Unidad didáctica 8} & Une fête & Unidad didáctica 7 & Correspondance \\
\hline & & Unidad didáctica 8 & Récit fantastique \\
\hline
\end{tabular}

Por tanto, cada libro pretende trabajar al menos un aspecto sociocultural en cada unidad didáctica, aunque en el desarrollo de cada una de ellas y tras el proceso de análisis hemos comprobado que en algunos casos esta intención se vea alterada por varios motivos que relatamos seguidamente.

Comenzando por el libro 'Vitamine 1', algunos de los temas que el libro presenta en el apartado de civilización no se corresponde con los elementos que el MCERL (2002) considera como socioculturales, tales como ocurre con 'les vêtements' en la unidad didáctica 5, cuya presentación de la ropa se limita al ámbito lingüístico sin haber ninguna presencia cultural. Por otro lado, algunos de estos temas indicados en el índice, no son desarrollados después de manera explícita en la unidad didáctica correspondiente, como es el caso de 'la situatión scolaire' en 
Análisis del componente cultural en libros de texto de francés destinados al tercer ciclo de Educación Primaria

ELISA GIL RUIZ y CARMEN SOTO PALlarés

la unidad didáctica 2. Esta unidad didáctica gira en torno a la temática mencionada en el título pero solo presenta contenidos gramaticales y lexicales, y no trata ninguna muestra cultural al respecto.

También, hay que destacar que en algunas unidades didácticas se encuentran muestras culturales que han sido analizadas pero que no se corresponde con los temas indicados en el índice del libro, tal como ocurre en la unidad didáctica 3, cuya temática de civilización es 'les prénoms usuels' y en nuestro análisis hemos encontrado muestras culturales que giran alrededor del bloque temático correspondiente a las convenciones sociales empleadas en los intercambios comunicativos.

En el libro 'Vitamine 2' ocurren algunos de los casos anteriormente descritos y localizados en el primer libro. Por ejemplo, existen algunos temas que el libro presenta en el apartado de 'Civilisation' que no se corresponden con los elementos que el MCERL (2002) considera como socioculturales, tales como 'l'écologie' en la unidad didáctica 5, que es más bien considerado un tema transversal. Por otro lado, en algunas unidades didácticas se encuentran muestras culturales que han sido analizadas aunque no se correspondan con los temas indicados en el índice del libro, tal como ocurre en la unidad didáctica 4, cuya temática cultural es 'les loisirs'. Sin embargo, en nuestro análisis hemos encontrado muestras culturales que giran en torno a la manera de desarrollar las relaciones personales entre adolescentes.

Basándonos en la evidencia material que revelan los casos comentados, podemos determinar que no existe una relación unívoca entre los temas culturales indicados en el índice y los realmente desarrollados. Esta falta de correspondencia entre los aspectos culturales destacados en el índice de contenidos y los realmente desarrollados en las unidades didácticas nos da muestras de una cierta descoordinación que, a nuestro juicio, se debe a que la editorial no ha planificado el desarrollo del componente cultural fuera de las secciones específicas establecidas.

Si tenemos en cuenta los bloques temáticos establecidos por el MCERL (2002), que han sido considerados como referentes para el análisis de la temática, el tema más abordado en las muestras culturales analizadas en estos dos libros es el 4, relativo a los valores, las creencias y las actitudes. Le sigue el tema 1, que corresponde a la vida diaria. También, el tema 3, que atañe a las relaciones personales y el tema 6 , relativo a las convenciones sociales, tiene una importante presencia en esta editorial, aunque en proporción menor a los anteriores bloques temáticos. 
El resto de temáticas consideradas por el MCERL (2002) ha sido tratado en las muestras analizadas, aunque de forma poco considerable, ya que su desarrollo no es comparable con los anteriores temas destacados. No obstante, cabe destacar que el tema 2, relativo a las condiciones de vida, no ha sido detectado en ninguna de las actividades analizadas en cada uno de los libros de esta editorial.

En cuanto al medio de transmisión del componente cultural, tenemos que destacar que el soporte texto es el mayoritariamente empleado. Sin embargo, no podemos dejar de destacar que los contenidos culturales no solo son transmitidos por medio de elementos verbales, sino que las fotos y las ilustraciones tienen una gran presencia en la difusión del componente cultural.

En lo relativo al tipo de actividades, el empleo de los diálogos es bastante acuciante en esta editorial, tanto en soporte texto como audio, ya que, este tipo de actividad permite no solo el aprendizaje de aspectos lingüísticos en un contexto determinado, sino también facilitan la transmisión de contenidos culturales, adaptados a una situación comunicativa concreta.

En este recorrido analítico merece igualmente comentario la utilización de los cómics, que por sí mismos son normalmente elementos que vehiculan bastantes contenidos culturales. Los que se encuentran presentes en esta editorial son elaborados por los autores del manual, por lo que no son materiales auténticos, pero presentan situaciones que se aproximan bastante a los intercambios comunicativos que se dan en la realidad, permitiendo así la observación de diversas convenciones sociales empleadas en distintas situaciones comunicativas.

Los fragmentos narrativos son bastante empleados, aunque de forma minoritaria a los dos tipos de actividades anteriores. Éstos son útiles para transmitir informaciones objetivas de la cultura extranjera, aunque, en la mayoría de los casos, éstos son asociados a elementos icónicos que facilitan la transmisión del significado.

Por otro lado, hay que remarcar que en todas las unidades didácticas se integran canciones y 'comptine', que son poesías infantiles muy repetitivas, pero la mayoría no han sido analizadas porque no son tradicionales, sino que han sido elaboradas por los autores del manual con una finalidad eminentemente lingüística. Las analizadas son las tradicionales que vehiculan aspectos culturales, las cuales son una minoría con respecto a la totalidad de las introducidas en cada uno de los libros. 
Análisis del componente cultural en libros de texto de francés destinados al tercer ciclo de Educación Primaria

Elisa GIL RUIZ y CARMEN SOTO PALLARÉS

Y como el elemento central de nuestro análisis son las actividades que incluyen muestras culturales, es necesario estimar de manera aproximada la cantidad de actividades que desarrollan aspectos culturales, teniendo en cuenta la totalidad de las existentes en el libro. Referimos a continuación nuestro análisis comparativo al respecto.

El número de actividades con presencia de componente cultural en ambos libros es similar. Por un lado, las actividades analizadas en el libro 'Vitamine 1', debido a su contenido cultural, son 27 de un total de unas 160 aproximadamente. Basándonos en este dato, podemos considerar que el componente cultural es desarrollado en este libro en un $16,88 \%$ del total de actividades, aproximadamente. Por otro lado, en el libro 'Vitamine 2' las actividades analizadas son 28 de un total de unas 160 actividades. Por tanto, y siempre de forma aproximada, las actividades que desarrollan el componente cultural suponen el 17,5\% del total de actividades de este libro de texto.

En otro orden de cosas cabe afirmar que, en referencia al tratamiento didáctico otorgado al componente cultural, hemos observado la introducción de rasgos metodológicos pertenecientes tanto al enfoque informativo como formativo. Igualmente hemos advertido que en el desarrollo de los tres suplementos culturales que presenta cada uno de los dos libros de esta editorial se transmite información objetiva y característica de la cultura francesa, de manera aislada al desarrollo lingüístico, ya que cada uno de estos suplementos se encuentra intercalado cada dos unidades didácticas. Además, estos suplementos estimulan también la separación entre los aspectos lingüísticos y los culturales, ya que son presentados como compartimentos estancos y aislados del desarrollo de las distintas unidades didácticas. Sin embargo, hemos de subrayar que hemos encontrado muestras culturales no solo en este apartado estanco o en los suplementos culturales, sino también se establecen contenidos culturales desarrollados e integrados con el aprendizaje lingüístico.

Un aspecto importante que, de forma general, hemos detectado es la intención de esta editorial de estimular actitudes positivas hacia la cultura extranjera, lo cual es fundamental al inicio del proceso de aprendizaje de una lengua extranjera. Así, se favorece la motivación del alumnado hacia la adquisición de dicha lengua, lo que facilita el desarrollo del aprendizaje y su implicación en dicho proceso.

Además, algunas de las muestras culturales analizadas han tratado de relativizar la cultura materna del alumnado, para que éstos consigan 
distanciarse de ella, y así afrontar el aprendizaje de la cultura extranjera desde otra perspectiva, en la que se haga favorable el desarrollo de una actitud de empatía y de sensibilidad cultural.

Sin embargo, no hemos encontrado en ninguno de los libros actividades que estimulen la comparación entre la cultura materna y la extranjera, ni la presentación de algún estereotipo sobre el que reflexionar para llegar a su superación y comprensión. Otra de las lagunas que hemos descubierto es la ausencia de actividades culturales que fomenten la adopción de las funciones propias de un intermediario cultural, aspecto que debe ser iniciado al comienzo del aprendizaje de una lengua extranjera, aunque en pequeña medida, debido al escaso nivel lingüístico del alumnado.

Otro aspecto destacable de este análisis es la deducción de que el tipo de cultura abordada en las muestras analizadas está en función del soporte empleado para vehicular el contenido cultural, pues, en efecto, en los suplementos culturales, en las fotos documentales y en los mapas se transmite una cultura formal, mientras que en los cómics, en los diálogos y en las ilustraciones se difunde una cultura no formal. Por otro lado, no hemos encontrado ninguna muestra cultural que vehicule contenidos pertenecientes a lo que hemos Ilamado la 'Kultura con K'.

En cuanto al tema del desarrollo de las competencias básicas, las muestras culturales detectadas estimulan, de forma mayoritaria, el desarrollo de la competencia cultural y artística, puesto que, hemos centrado nuestra atención en las actividades que divulgan el componente cultural. En segundo lugar, nos encontramos con la preponderancia de la competencia en comunicación lingüística seguida por la competencia social y ciudadana en el desarrollo de las actividades culturales analizadas. La primera es desarrollada en todas las actividades culturales que vehiculan elementos verbales, ya sean escritos u orales. La segunda suele ser estimulada, sobre todo, en los cómics, en los diálogos y en algunas actividades del soporte icónico.

Además, en las muestras analizadas, hemos detectado la presencia de otras competencias, aunque éstas representan una proporción bastante inferior. Éste es el caso de la competencia en el conocimiento y la integración con el mundo físico, desarrollada, sobre todo, por medio de los mapas. En cuanto al tratamiento de la información y competencia digital, esta capacidad conjunta es impulsada en el desarrollo del componente cultural cuando el libro establece direcciones Web para estimular en 
Análisis del componente cultural en libros de texto de francés destinados al tercer ciclo de Educación Primaria

ELISA GIL RUIZ y CARMEN SOto Pallarés

el alumnado la ampliación de los contenidos trabajados. Ejemplo de esta incardinación entre competencias es igualmente el impulso que las actividades culturales estudiadas otorgan a la autonomía e iniciativa personal.

Finalmente, es necesario indicar que en ninguno de los dos libros de esta editorial, las actividades culturales han estimulado el desarrollo ni de la competencia matemática ni de la competencia para aprender a aprender.

\section{Conclusiones}

Tras el análisis de los resultados obtenidos, podemos concluir que la editorial Santillana ha considerado el componente cultural en el desarrollo de los libros de francés destinados al tercer ciclo de Educación Primaria.

En esta editorial, los elementos culturales han sido desarrollados, en gran medida, en un apartado aislado del aprendizaje lingüístico, Ilamado 'civilización'. Además, cada dos unidades didácticas, la editorial introduce un apartado de cultura con el nombre de 'France Magasine', que refuerza todavía más el desarrollo cultural en un compartimento estanco con respecto al aprendizaje lingüístico.

De hecho, la existencia de estas dos secciones culturales no se corresponde con las orientaciones de los actuales enfoques metodológicos, los cuales promulgan la integración de los contenidos lingüísticos y culturales en la adquisición de una lengua extranjera, para estimular así el desarrollo de la competencia comunicativa.

Asimismo, los aspectos culturales no se equiparan en número al resto de actividades en los libros de esta editorial, los cuales deberían tener una mayor presencia para favorecer así la integración de la lengua y la cultura. De esta manera, el componente cultural debería estar representado en cada libro de texto analizado en una proporción equiparable a la presencia de los contenidos lingüísticos. También, es importante destacar la necesidad de variar la temática abordada en las actividades culturales para favorecer el acceso a un amplio abanico de contenidos que permita al alumno ampliar su visión de la cultura extranjera, siempre relacionándola con su cultura materna.

Finalmente, es importante ofrecer una variedad de registros culturales, presentando aspectos pertenecientes tanto a una cultura formal, no 
formal como a la "Kultura con K". Sin embargo, la editorial analizada no ha incluido ninguna actividad en la que se da a conocer la "Kultura con K", sino que las actividades culturales han desarrollado contenidos culturales, tanto formales como no formales.

Como propuesta de mejora, proponemos la necesaria revisión de la inclusión del componente cultural en los libros de francés de la editorial Santillana, con el objetivo de incrementar su presencia y favorecer así el desarrollo de la competencia comunicativa del alumnado.

\section{Referencias bibliográficas}

Areizaga, E. (2002). El componente cultural en la enseñanza de lenguas: elementos para el análisis y la evaluación del material didáctico. Cultura y Educación, 14 (2), 161 175.

Areizaga, E., Gómez, I. e Ibarra, E. (2005). El componente cultural en la enseñanza de lenguas como línea de investigación. Revista de Psicodidáctica, 10 (2), 27-46.

Consejo de Europa (2002). Marco Común Europeo de Referencia para las lenguas: aprendizaje, enseñanza, evaluación. Madrid: MEC, Instituto Cervantes y Editorial Anaya.

Guillén Díaz, C. (2002). La dimensión cultural de la enseñanza /aprendizaje de lenguas extranjeras. En C. Guillén Díaz, Lenguas para abrir caminos. (193-226). Madrid: Ministerio de Educación, Cultura y Deportes

Ley orgánica 2/2006, de 3 de mayo, de educación. BOE, núm. 106, de 4 de mayo 2006. (LOE).

Martín Nolla, C. y Pastor D.D. (2009a). Vitamine 1. Madrid: Santillana.

Martín Nolla, C. y Pastor D.D. (2009b). Vitamine 2. Madrid: Santillana.

Miquel, L y Sans, N. (1992). El componente cultura I: un ingrediente más en las clases de lengua. Cable, 9, 15-21. 
